\title{
Portrait de la formation dans la Vallée de l'aluminium
}

\author{
Diane Brassard, M.Ps., M.Sc. \\ Centre de recherche sur le développement territorial (CRDT) de l'UQAC
}

\section{Introduction}

Nous présentons ici le portrait de l'offre de formation dans le domaine de l'aluminium dans la Région du Saguenay - Lac-Saint-Jean, aussi appelée Vallée de l'aluminium, que ce soit la formation universitaire à l'Université du Québec à Chicoutimi (UQAC), la formation technique dans les collèges d'enseignement général et professionnel (CEGEPS) et la formation professionnelle dans les centres de formation professionnelle (CFP) des commissions scolaires (C.S.) régionales, sans oublier la formation continue offerte par les Services d'aide aux entreprises (SAE) associés aux CEGEPS et aux C.S. de la région. Nous jetons aussi un coup d'œil sur la plateforme d'apprentissage en ligne dédiée à l'aluminium développée, entre autres, par l'UQAC et le Centre québécois de recherche et développement de l'aluminium (CQRDA). Une des priorités du CQRDA est la formation et le perfectionnement des différentes catégories de personnel de l'industrie de l'aluminium. Plusieurs organismes régionaux encouragent la formation dans le domaine de l'aluminium en mettant sur pied des concours auprès des jeunes et en accordant des bourses d'études aux étudiants universitaires. Nous décrirons brièvement le rôle du Comité sectoriel de la main-d'œuvre en métallurgie qui tente de faire le lien entre les établissements de formation et les besoins de main-d'œuvre des entreprises petites et grandes. En conclusion, nous tenterons de voir si l'offre de formation correspond bien aux besoins des entreprises régionales. Finalement, nous examinerons quelques pistes afin d'améliorer l'offre de formation au Saguenay - Lac-Saint-Jean dans le futur.

\section{Formation universitaire à l'Université du Québec à Chicoutimi (UQAC) ${ }^{1}$}

En lien direct avec le domaine de l'aluminium, l'UQAC offre un baccalauréat en ingénierie de l'aluminium. Pour répondre aux besoins du milieu, elle offre également d'autres formations connexes, tant au niveau du baccalauréat, de la maîtrise, que du doctorat. Nous ajouterons un mot sur l'avenir de la formation universitaire dans le domaine de l'aluminium.

\subsection{Formation spécifique au domaine de l'aluminium}

Durant les quatre années que dure le programme de baccalauréat en ingénierie de l'aluminium, l'étudiant approfondit «les sciences de base de l'ingénierie, les mathématiques appliquées et l'informatique ». Afin de répondre aux besoins des entreprises et de l'industrie, il développe aussi ses compétences en conception de projets. Deux concentrations sont possibles : "production industrielle » et «métallurgie et transformation ». L'enseignement de cette dernière se fait «à partir des disciplines de base de la métallurgie et du génie mécanique, toutes orientées vers l'aluminium ». On y aborde notamment «l'étude de la mise en forme, des traitements thermiques et de surface, des méthodes d'assemblage connues et émer- 
gentes, ainsi que des nouveaux matériaux et des alliages conçus à partir du métal gris ». «Le diplômé de ce programme peut orienter sa carrière selon un éventail de possibilités qui comprend, entre autres, la conception technique d'appareillages ou d'ouvrages, la gestion et la direction de projets d'ingénierie, le génie-conseil, la représentation technique, le contrôle de procédés et la supervision d'opérations de production de biens et de services ou d'exploitation de ressources. Toutes ces possibilités se retrouvent aussi bien dans les grandes, moyennes et petites industries que dans les organismes publics et parapublics et les firmes de génie-conseil ».

\section{La présence de ces nouvelles structures} de recherche de haut niveau sur le campus de l'UQAC empêchera peut-être l'exode de nos jeunes ingénieurs vers les grands centres

À la maîtrise et au doctorat, l'UQAC offre aussi des programmes en ingénierie qui, sans être spécifiques, ouvrent des portes vers le domaine de l'aluminium. Mentionnons que le Centre universitaire de recherche sur l'aluminium (CURAL) accueille annuellement une quarantaine d'étudiants à la maîtrise et au doctorat dans ses nouveaux laboratoires (le Pavillon Rio Tinto - Alcan) supervisés par une trentaine de chercheurs et de professeurs. Deux axes de recherche sont priorisés au CURAL: la transformation de l'aluminium (produits finis et semi-finis) et la transformation de la bauxite.

\subsection{Autres formations connexes}

Sans être spécifiques au domaine de l'aluminium, l'UQAC offre des formations connexes. Au niveau du baccalauréat, les programmes suivants sont recherchés par les entreprises régionales: génie informatique, génie mécanique et génie électrique.

\subsection{Avenir de la formation universitaire}

La récente mise en place du Pavillon Rio Tinto - Alcan du CURAL regroupe toutes les forces vives en matière de recherche de 1'UQAC. Situé à deux pas, il est en lien étroit avec les Centre des technologies de l'aluminium (CTA). Ceci ne pourra qu'encourager les actuels étudiants du baccalauréat en ingénierie de l'aluminium à poursuivre ici même leurs études supérieures entourés des meilleurs chercheurs dans le monde intéressés au développement de nouvelles technologies de pointe dans le domaine de l'aluminium. La présence de ces nouvelles structures de recherche de haut niveau sur le campus de l'UQAC empêchera peut-être l'exode de nos jeunes ingénieurs vers les grands centres.

\section{Formation technique dans les collèges d'enseignement général et professionnel $(\text { CEGEPS })^{2}$}

Trois des quatre CEGEPS de la région sont impliqués au niveau de la formation technique dans le domaine de l'aluminium: le CEGEP de Chicoutimi, celui de Jonquière et le Collège d'Alma. Certaines des formations offertes sont spécifiques au domaine de l'aluminium, alors que d'autres sont connexes. Nous ajoutons également un mot sur l'avenir de la formation collégiale selon la Fédération des CEGEPS du Québec.

\subsection{Formations spécifiques au domaine de l'aluminium}

2.1.1 Attestation d'études collégiales (AEC) en technologie de la transformation de l'aluminium 
Formation technique d'une durée de 1830 heures (dont 240 heures de stage) offerte dans trois CEGEPS de la région: Chicoutimi, Jonquière et Alma. Les objectifs poursuivis ici sont la formation de «professionnels de la transformation de l'aluminium aptes à travailler dans les entreprises de transformation de l'aluminium à partir des procédés de moulage, d'extrusion, de laminage, de mise en forme à chaud et à froid, ainsi que celle de finition des surfaces. Le programme couvre la production de lingots coulés en semi-continu à refroidissement direct jusqu'à la finition de pièces de deuxième et troisième transformation ».

2.1.2 Diplôme d'études collégiales (DEC) en technologie du génie métallurgique, option procédés de transformation

Formation technique d'une durée de 2580 heures (dont 1920 heures spécifiques aux procédés de transformation) est offerte conjointement par le Collège d'Alma (première année) et le CEGEP de Chicoutimi (deuxième et troisième années). Elle vise «la formation de techniciens en métallurgie aptes à travailler dans diverses catégories d'entreprises : les alumineries, les aciéries, les fonderies, les entreprises de laminage, de moulage et d'extrusion, les forges, les entreprises d'atomisation, d'électrolyse, d'électroplacage et de revêtement ». Les personnes qui obtiennent ce diplôme pourront " analyser des matériaux, résoudre des problèmes de procédés ou améliorer ces derniers, effectuer des activités de formation, participer à l'élaboration et à l'implantation de méthodes de travail, effectuer des activités

Sans être spécifiques au domaine de l'aluminium, certaines formations techniques offertes dans la région répondent aux besoins des employeurs de ce secteur relatives à la recherche et au développement de même que des activités de soutien technique ».

\subsection{Autres formations connexes}

Sans être spécifiques au domaine de l'aluminium, certaines formations techniques offertes dans la région répondent aux besoins des employeurs de ce secteur. Certaines formations prennent la forme d'alternance travail-études (ATE), où le milieu de travail est au cœur de la formation; l'expérience de travail, la connaissance des exigences en milieu de travail et la possibilité d'attirer l'attention de l'employeur donnent à l'élève une longueur d'avance pour l'obtention d'un emploi à la fin de ses études. D'autres formations permettent un passage harmonieux entre le CEGEP et l'université; ce sont les DEC-BAC. Voici les formations que nous avons retenues du «Guide d'admission 2008$2009 »$ préparé par le regroupement des CEGEPS du Saguenay - Lac-Saint-Jean : technique du génie chimique (ATE - CEGEP de Jonquière); technique du génie mécanique (DEC-BAC - CEGEP de Jonquière); technique de laboratoire (chimie analytique) (ATE - CEGEP de Jonquière); technique de l'électronique industrielle (ATE et DEC-BAC - CEGEPS de Chicoutimi et de Jonquière).

\subsection{Avenir de la formation collégiale}

Dans un communiqué de presse daté du 28 novembre 2007, la Fédération des CEGEPS du Québec réclame «un élargissement de l'accès à des services à temps plein ou à temps partiel pour tous les adultes, à travers un financement plus souple». (...) «Le tout passe nécessairement par une relance de la formation à temps partiel, dans les programmes d'AEC et de DEC et sous la forme de cours « hors programme », une formule qui attire la majorité des adultes inscrits en formation continue dans les autres provinces canadiennes. » (...) « Le développement des services de reconnaissance des acquis et des 
compétences, ainsi que la diversification de l'offre de formation manquante, constitue d'autres solutions à mettre en œuvre rapidement ». (...) «Le transfert au Québec, en 2008-2009, d'une somme de 120M\$ issue du dernier budget fédéral et destiné à soutenir la formation liée à l'emploi devra contribuer à élargir l'accès des adultes à la formation offerte au CEGEP ».

\section{Formation professionnelle dans les commissions scolaires (C.S.) ${ }^{3}$}

Trois C.S. de la région participent activement à la formation des futurs travailleurs dans le secteur de l'aluminium, soit la C.S. des Rives- nécessaires pour exécuter les travaux préparatoires au soudagemontage, réaliser des projets de montage et de soudage d'éléments de du-Saguenay (le CFP en métallurgie multiservices de Chicoutimi - CFPMM et le CFP de la Baie), la C.S. du Lac-Saint-Jean (le CFP d'Alma - Pavillon Bégin) et la C.S. du Pays-des-Bleuets (CFP de DolbeauMistassini). Deux types de formation sont spécifiques au domaine de l'aluminium, alors que d'autres sont connexes. Nous ajoutons quelques mots sur l'avenir de la formation professionnelle en ce domaine dans la région.

3.1 Formations spécifiques au domaine de l'aluminium

3.1.1 Attestation d'études professionnelles (AEP) en soudage des alliages d'aluminium

Formation professionnelle d'une durée de 630 heures qui vise «l'acquisition des compétences nécessaires à la réalisation d'assemblage et à l'exécution du soudage de produits en alliage d'aluminium avec les procédés GTAW, GTAW-P, GMAW et GMAW-P ». Cette formation est offerte au CFPMM de Chicoutimi.

\subsubsection{Diplôme d'études professionnelles (DEP) en soudage-montage}

Formation professionnelle d'une durée de 1800 heures qui a pour objectifs «d'acquérir les connaissances, les habiletés et les attitudes base, d'éléments de structure, des projets de montage industriels et de structures métalliques avec différents procédés de soudage (SMAW, GTAW, GMAW, FCAW, SAW, RW, PAW), lire des plans industriels et interpréter des devis, ainsi que des procédures de soudage ». Elle est offerte au CFPMM de Chicoutimi, au CFP d'Alma et au CFP de Dolbeau-Mistassini.

\subsection{Autres formations connexes}

D'autres types de formation professionnelle sont également en demande dans le secteur de l'aluminium, notamment les techniques d'usinage (DEP - CFP d'Alma), l'usinage sur machines-outils à commandes numériques (ASP - CFP d'Alma - Pavillon Auger), soudage haute-pression (ASP - CFP en métallurgie et multiservices), fonderie (DEP CFP la Baie).

\subsection{Avenir de la formation professionnelle}

Le 21 juillet 2007, la C.S. des Rives-duSaguenay annonçait dans le journal «le Quotidien » un projet très novateur sous forme de partenariat public-privé dans le domaine de la formation professionnelle. La C.S. et une quarantaine de partenaires impliqués dans le domaine de l'aluminium ont en effet décidé d'investir plus de trois millions de dollars 
(dont un million de dollars provenant du secteur privé) dans la construction de la nouvelle aile exclusivement réservée à l'aluminium qui sera réalisée au CFP Laure-
Conan. Les nouveaux locaux du CFPMM de Chicoutimi seront disponibles en septembre 2009.

\section{Formation continue offerte par les Services d'aide aux entreprises (SAE) ${ }^{4}$}

«Aujourd'hui confrontées à des situations nouvelles, à la mondialisation des marchés et aux fluctuations de l'économie, les entreprises doivent s'adapter pour rester compétitives. Pour les guider dans ce parcours vers un développement optimal, un partenaire de choix s'impose : le réseau public des SAE du Québec. Les SAE des CEGEPS et des C.S. offrent aux entreprises et organismes des services de formation continue et de perfectionnement de la main-d'œuvre dans un grand nombre de secteurs d'activité (dont la métallurgie). Grâce au partenariat entre les milieux scolaire, industriel et social, les SAE participent activement à la croissance socioéconomique régionale. Ils entrevoient et soutiennent l'évolution technologique et organisationnelle des entreprises. Les SAE mettent en commun leur savoir-faire, leurs connaissances et leur expérience, formant ainsi un réseau fort et polyvalent, au service des individus, des entreprises et des organisations. De plus, le réseau couvre l'ensemble du territoire québécois. »

Les quatre CEGEPS (Alma, Chicoutimi, Jonquière, Saint-Félicien) et les quatre C.S. (Jonquière, Rives-du-Saguenay, Lac-SaintJean et Pays-des-Bleuets) du Saguenay - LacSaint-Jean offrent des SAE en matière de formation continue. Ces formations, taillées sur mesure, répondent aux besoins spécifiques des entreprises du secteur de l'aluminium, peu importe leur taille, améliorant ainsi leur performance. Voyons quelques exemples.

$\mathrm{Au}$ CEGEP de Chicoutimi, le Centre de formation continue «Humanis» offre aux organisations qui désirent améliorer leurs performances des services de formation et de consultation dans le domaine des techniques physiques à un coût raisonnable et avec des méthodes d'enseignement adaptées. Dans le secteur de l'industrie de la métallurgie, «Humanis» offre du soutien dans la résolution des problèmes techniques et dans l'optimisation des opérations (procédés et techniques de transformation des métaux, contrôle des procédés de production et de fabrication, contrôle des techniques et procédés de parachèvement).

Les Services de formation continue du CEGEP de Jonquière a développé une expertise diversifiée en consultation et en formation sur mesure dans le domaine des techniques physiques, entre autres. On intervient notamment dans les domaines suivants: métallurgie et transformation de l'aluminium et production du métal primaire (électrolyse). Le programme électrolyse industrielle (AEC), d'une durée de 900 heures, permet aux étudiants d'intégrer le milieu industriel de production de métaux légers dans le secteur de l'électrolyse. Le programme est conçu en fonction des besoins des industries de l'électrolyse (aluminium, magnésium, zinc et électro-placage).

Le Service de formation continue du Collège d'Alma offre le programme des technologies de la transformation de l'aluminium (ETA) d'une durée de 1560 heures. "Ce programme vise la formation des professionnels de la transformation de l'aluminium aptes à travailler dans les entreprises de transformation de l'aluminium à partir des procédés de moulage, d'extrusion, de 
laminage, de mise en forme à chaud et à froid, ainsi que celles de finition ou de traitement de surface ».

Le Centre des services aux entreprises de la C.S. de la Jonquière a développé un "programme complet sur les procédés de revêtement sur divers métaux et plus spécifiquement sur l'aluminium ». Des spécialistes peuvent intervenir en soutien technique et en diffusion de formation dans plusieurs domaines : préparation des surfaces et des pièces; application de peinture liquide par pulvérisation, application de peinture en poudre, conception et utilisation d'outils d'accrochage, opération de décapage, contrôle qualité basé sur les spécifications SSPC.

Le Service aux entreprises de la C.S. des Rives-du-Saguenay, en lien avec le CFPMM de Chicoutimi, offre plusieurs formations qualifiantes aux entreprises, dont la certification des compagnies de soudage par fusion des structures d'aluminium et la qualification des soudeurs (structures en aluminium). D'autres services sont également disponibles : consultation, analyse des besoins, profil des compétences, plan de formation, reconnaissance des acquis, tests de classement, sélection de personnel, formation des formateurs, traduction technique, conception et réalisation de pièces prototypes.

Le réseau public des services aux entreprises (SAE) du Québec a consulté ses membres en 2003 afin de voir comment chaque région pourrait mieux travailler en collaboration et en partenariat pour être une force régionale et aider les organisations à faire face aux défis du futur. Voici les constats des répondants de la Vallée de l'aluminium. Les entreprises n'étaient pas encore en manque de maind'œuvre à l'époque, mais l'exode des jeunes vers les grands centres, plus particulièrement les plus scolarisés, posera sous peu un problème de taille aux industries régionales. Il faudra, entre autres, préparer la relève en fonction des nouveaux besoins des entreprises.

\section{Plateforme d'apprentissage en ligne dédiée à l'aluminium ${ }^{5}$}

Après quatre années d'efforts concertés, $M$. Michel Belley, recteur de l'UQAC, et M. Damien Ferland, directeur du Service de formation continue de l'UQAC, lançaient en novembre 2005 la plateforme d'apprentissage en ligne entièrement dédiée à l'aluminium avec ses nombreux partenaires: le CQRDA, Valorisation-recherche Québec et l'Agence universitaire de la francophonie. Ce portail électronique, dénommé «CentrAL », centralise l'ensemble des savoirs détenus au sein de l'industrie internationale de l'aluminium. Le volet formation comprend trois sections : formation sur mesure, exercices et évaluation, suivi détaillé.

\section{«CentrAL $»$, centralise l'ensemble des savoirs détenus au sein de l'industrie internationale de l'aluminium.}

\section{Formation et perfectionnement des différentes catégories de personnel de l'industrie de l'aluminium par le Centre québécois de recherche et développement de l'aluminium $(\text { CQRDA })^{6}$}

Entre juin 2002 et décembre 2006, le CQRDA a accepté onze projets en lien avec la formation et le perfectionnement des différentes catégories de personnel de l'industrie de l'aluminium, dont cinq au Saguenay - Lac-Saint-Jean: Formation spécialisée en moulage permanent à gravité pour le Centre de formation et de 
développement en métallurgie (CFDM) de la Baie; Programme relatif à l'utilisation de l'aluminium dans la réalisation d'un châssis d'automobile en développant les procédés de fabrication pour les SAE-UQAC; Création d'un logiciel éducatif dans le domaine du soudage et création d'un simulateur de soudage à partir d'un jeu éducatif pour 123 Certification Inc.; Édition d'un guide de com-

\section{Concours auprès des jeunes et bourses talents dans le secteur de l'aluminium}

Pour répondre aux besoins futurs de maind'œuvre dans le domaine de l'aluminium et faire en sorte que nos entreprises, petites et grandes, soient plus compétitives sur les marchés internationaux grâce à leurs innovations, plusieurs organismes de la région mettent sur pied des concours auprès des jeunes et offrent des bourses d'études universitaires.

En mars 2008, la seconde édition du concours de création « Rêver l'aluminium » est lancée à plus de 560 élèves de secondaire III dans le cadre de leur cours de science afin d'imaginer une pédale de vélo révolutionnaire moulée en aluminium. Plusieurs partenaires soutiennent ce défi via une simulation d'entreprise où chacun joue un rôle actif: la C.S. du LacSaint-Jean, le Service de formation continue «Humanis » du CEGEP de Chicoutimi, la Société de la Vallée de l'aluminium (SVA), la Fonderie Bleu Matière, Cycles Devinci et le Groupe génitique. Le tout a pour but de sensibiliser les élèves à l'industrie de l'aluminium, notre créneau d'excellence régional. Le prix est une bicyclette Devinci munie de la pédale gagnante.

Le concours «Génie-Al » du CQRDA soutient la valorisation de la transformation de l'aluminium par les étudiants de premier cycle universitaire en génie qui présentent un projet menant à la conception d'un produit en mercialisation de produits industriels pour les Presses de l'aluminium (PRAL).

En janvier 2007, en compagnie de ses partenaires et via sa maison d'édition (PRAL), le CQRDA a aussi rendu public le «Guide de soudage des alliages d'aluminium» de l'auteur Julien Lapointe et les nouvelles versions françaises des «Modules de formation - le soudage de l'aluminium ».

\section{d'études favorisant l'éclosion de nouveaux} aluminium dans le cadre d'une compétition universitaire (trois prix totalisant $6000 \$$ ). Le CQRDA présente également le concours «PARU» (programme d'appui à la recherche universitaire) qui aide financièrement les chercheurs universitaires qui encadrent des étudiants à la maîtrise $(5000$ ) ou au doctorat (10 000\$) dont les sujets de recherche cadrent avec la mission du CQRDA.

Voici aussi quelques exemples de bourses d'études offertes dans la région. Les compagnies Spectube, CROI, Cycles Devinci et Alouette, en collaboration avec le CTA, le CURAL de l'UQAC et l'Université Laval, offrent une bourse à la maitrise de $18000 \$$ afin de concevoir des pièces via un enchaînement de procédés de mise en forme de tubes d'aluminium.

Quatre bourses «Relève Alcan» de $3000 \$$ chacune (plus un stage rémunéré durant l'été 2008) visent les étudiants de premier cycle d'horizons divers, dont le génie mécaniqueélectrique et l'ingénierie de l'aluminium. La «Bourse d'études supérieures Alcan » d'une valeur de $18000 \$$ est également offerte aux étudiants de maitrise ou doctorat dans une discipline appartenant aux sciences pures et appliquées reliée aux domaines d'activités de l'entreprise (matières premières, électrolyse, métallurgie de l'aluminium, son utilisation, environnement, etc.). 


\section{Comité sectoriel de la main-d'œuvre en métallurgie}

«Le Comité sectoriel de la main-d'œuvre en métallurgie est une organisation paritaire à but non lucratif qui regroupe des représentants d'entreprises et de syndicats de travailleurs de l'industrie métallurgique de première transformation. Sa mission est de répondre aux besoins du secteur en matière d'emploi, de formation et d'adaptation de la main-d'œuvre. Le comité investit prioritairement ses efforts dans la recherche et la préparation d'une relève qualifiée et apte à occuper les nouveaux emplois qui deviendront disponibles dans les années à venir. » Dans le domaine de l'aluminium, les organismes régionaux qui participent à ce comité proviennent d'une dizaine d'entreprises du milieu (cinq usines du Groupe Rio Tinto Alcan et cinq PME), d'organisations diverses (SVA, Réseau TransAl, AAC, CSN, FTQ), de groupes de recherche (CQRDA, CURAL, CTA), du monde de la formation professionnelle (les quatre commissions scolaires régionales) ou du secteur du financement (MDEIE, association des CLD).

\section{Conclusion}

L'offre de formation dans le domaine de l'aluminium est vaste et très variée dans la Vallée de l'aluminium, tant au niveau universitaire, collégial et professionnel, qu'en matière de formation

$L$ 'offre de formation dans le domaine de l'aluminium est vaste et très variée dans la Vallée de l'aluminium continue. Mais correspond-elle aux besoins des entreprises régionales, à savoir la grande entreprise Rio Tinto - Alcan principalement axée sur la première transformation de l'aluminium et la trentaine de PME particulièrement intéressées à la deuxième ou la troisième transformation des produits de l'aluminium?

Le virage de Rio Tinto - Alcan vers la nouvelle technologie AP-50 pour la première transformation de l'aluminium nécessitera certainement une mise à jour des connaissances de ses employés actuels à l'Usine de Jonquière et des futurs travailleurs qui devront bientôt prendre la relève de cette main-d'œuvre vieillissante. A nulle part dans la région on ne parle encore de former ce type de travailleur. Il faudra que rapidement des ponts soient établis entre la grande entreprise et les institutions de formation collégiale et professionnelle afin de mettre en place de nouvelles formations axées sur la technologie AP-50. La place de la formation continue adaptée aux besoins des travailleurs d'usine est aussi à repenser: plus de souplesse dans les horaires et formation davantage ciblée avec des cours sur mesure, hors-programme. Par contre, les investissements récents dans le Pavillon Rio Tinto Alcan du CURAL et les programmes de bourses universitaires Alcan permettront sans doute d'accroître le nombre d'étudiants universitaires (ingénieurs et chercheurs de toutes sortes) dans la région.

Quant aux PME régionales, elles ont déjà fait les premiers pas en investissant près d'un million de dollars dans la construction d'une nouvelle aile exclusivement réservée à l'aluminium au CFP Laure-Conan de la C.S. des Rives-du-Saguenay qui sera disponible en septembre 2009. Ce nouveau partenariat public-privé est très innovateur et permettra aux PME d'avoir rapidement accès à une main-d'œuvre professionnelle qualifiée. De plus, les liens développés au cours des dernières années entre les PME et les divers centres de recherche de la région, notamment 
le CQRDA, le CTA et le CHT, semblent très prometteurs au plan de la formation vers de nouvelles technologies de pointe plus performantes. Le seul problème à régler est la rétention de leurs employés bien formés qui, dès qu'ils en auront la chance, s'en iront vers la grande entreprise qui offre des salaires plus avantageux que les PME.

Une des quatre recommandations de la «Carte routière technologique canadienne de la transformation de l'aluminium » (2006) est la promotion de la formation continue sur l'aluminium. Plusieurs opportunités en ce domaine pour le futur y sont mentionnées : « les transports, la construction, le moulage, le formage, l'assemblage, le traitement des surfaces et l'usinage ». L'offre de formation dans la région va un peu dans ce sens, notamment en ce qui a trait au moulage, au traitement des surfaces et à l'usinage.

\section{Notes et références}

1 L'essentiel des informations concernant le programme de baccalauréat en ingénierie de l'aluminium sont tirées du site internet suivant : http://wprod6.uqac.ca/programmes/desc_tap.html?code=7338.

2 L'essentiel des informations concernant la formation professionnelle et technique offerte dans la région dans le secteur de l'aluminium (CEGEP et CFP des C.S.) sont tirées de l'inforoute FPT disponible sur le site internet suivant : http://www.inforoutefpt.org

3 Voir la note 2 précédente.

4 Nous tirons nos renseignements sur les services d'aide aux entreprises (SAE) des sites internet suivants : http://www.inforoutefpt.org ; ceux des cégeps et des commissions scolaires mentionnés.

5 Communiqué de presse intitulé «L'apprentissage en ligne dédié à l'industrie de l'aluminium : un avenir assuré au niveau international» en date du 24 novembre 2005 disponible sur le site internet suivant: http://www.uqac.ca/medias/communique/2005/2005_29/php

6 «Plan quinquennal 2007-2012» du CQRDA disponible sur le site internet suivant: http://www.cqrda.qc.ca/publications/document/plan_quinquennal.pdf

\section{LEXIQUE :}

AAC : Association de l'aluminium du Canada

AEC : Attestation d'études collégiales

AEP : Attestation d'études professionnelles

ATE : Alternance travail-études

BAC : Baccalauréat

CFC : Centre de formation continue

CFP : Centre de formation professionnelle

CFPMM : Centre de formation professionnelle en métallurgie multiservices

CHT : Centre de haute technologie

CLD : Centre local de développement

CQRDA: Centre québécois de recherche et développement de l'aluminium

C.S. : Commission scolaire

CTA : Centre des technologies de l'aluminium
CURAL: Centre universitaire de recherche sur l'aluminium

DEC : Diplôme d'études collégiales

DEC-BAC: Diplôme d'études collégiales baccalauréat

FPT : Formation professionnelle et technique

MDEIE : Ministère du développement économique, innovation et exportation

PARU : Programme d'appui à la recherche universitaire

PME : Petites et moyennes entreprises

PRAL : Presses de l'aluminium

SAE : Services d'aide aux entreprises

SVA : Société de la Vallée de l'aluminium

UQAC : Université du Québec à Chicoutimi 


\section{PUBLICITIÉS}

CEE

MBA

MGO

BAA 